\title{
A note on nilpotency in a Left Goldie near-ring
}

\author{
K.C.Chowdhury \\ Department of Mathematics Gauhati University-781014, Assam,India
}

\begin{abstract}
In this paper we present an important result that a nil subnear-ring of a semiprime strictly left Goldie near-ring is nilpotent. It is to be noted that the essentiality of left near-ring subgroup here, arises as crucial from it's feeble nature. In contrast to such a result in ring theory, the crucial role played by substructures already mentioned appears here with very fascinating distinctiveness.
\end{abstract}

Keywords: near-ring, semiprime, nil subring, nilpotent subring, sequentially nilpotent, Goldie near-ring MR 2010 subject classification: 16Y30,16Pxx, 16P60,16U20

\section{Introduction}

Chowdhury et al [1] introduced the notions of a Goldie near-ring as well as that of a Goldie module $[2,3]$ as two way generalizations of so-called Goldie ring - an exposition of A.W. Goldie through his classics,- a part of his thorough study of the structure of prime rings under ascending chain conditions [10] and semiprime rings with maximum conditions [11]. We discussed various aspects of a Goldie near-ring and of a Goldie module including the near-ring of quotients and its possible descending chain condition and decomposition of the zero of a Goldie module [2,3], an analogous of Artin-Rees theorem [2]. Also we delve into Some Aspects of Artinian (Noetherian) Part of a Goldie Ring and its Topological Relevance (8) as well as Wreath Sum of Nearrings and Near-ring Groups with Goldie structures (9)). It is easy to see that a nilpotent subring of a ring is necessarily nil. But converse is not true, however, we see that [12] Goldie character in a ring draw attention in its favor!.We here prove this interesting standard problem in a near-ring with Goldie characteristics taking into consideration various aspects of large or essential characters of its subalgebraic structures with proper justification. Moreover, in this connection, it would not be irrelevant to mention author's another new notion, what may be called the notion of a nilpotent module-element or a nilpotent $\mathrm{N}$-group element [7] together with $a$ nil or a nilpotent submodule, or an $N$ subgroup etc.

\section{Preliminaries}

For the sake of completeness we would like to begin our discussion with the definition of a right near-ring $(N,+,$.$) - an algebraic structure consisting of a non-empty set N$ equipped with two binary operations viz., addition (+) and multiplication (.), where the first one makes $\mathrm{N}$ - a group (not necessarily abelian) and the second one a semigroup with the one-way distributive law, viz. $(\mathrm{a}+\mathrm{b}) \mathrm{c}=\mathrm{ac}+\mathrm{bc}$, for $\mathrm{a}, \mathrm{b}, \mathrm{c} \in N$ For other relevant information regarding near-ring preliminaries we would like to refer Pilz [13].

Throughout this paper $N$ will mean a right near-ring with unity (zero symmetric) unless otherwise specified.

\subsection{Definitions:}

2.1.1 An element $\mathrm{a} \in N$ is nilpotent if there is a positive integer $\mathrm{t}$ such that $\mathrm{a}^{\mathrm{t}}=0, \mathrm{a}^{\mathrm{t}-1} \neq 0$.

2.1.2. A subnear-ring is nil if each element of the corresponding set is nilpotent.

2.1.3. A subnear-ring $I$ is nilpotent if there is a positive integer $\mathrm{t}$ such that $\mathrm{I}^{\mathrm{t}}=0, \mathrm{I}^{\mathrm{t}-1} \neq 0$, (in the sense $i_{1} \cdot i_{2} \ldots i_{t}=0$, for $\mathrm{i}_{\mathrm{j}} \in I$ and $i_{1}, i_{2} \ldots i_{t-1} \neq 0$, for some $\mathrm{i}_{\mathrm{j}} \in I$ )

Clearly, a nilpotent subnear-ring is nil but the converse is not true. For the converse, that is a nilpotent subring is nilpotent, we'll deal with so called sequentially nilpotent (or s-nilpotent) notion.

We note the following: the above situation is dealt with the following definition that would lead us to our expected goal.

An element a $(\epsilon I)$ is sequentially nilpotent (s-nilpotent) if for some positive integer $\mathrm{k}$, we have $\left(a_{i} \in I\right)$

$a_{1} \cdot a_{2} \ldots a_{k}=0,\left(\mathrm{a}_{1}=\mathrm{a}\right)$. So if an element a $\left(\mathrm{a}_{1}=\mathrm{a}\right)$ is s-nilpotent, then for some

$(a=) a_{1}, a_{2}, \ldots, a_{k} \in I, a_{1} \cdot a_{2} \ldots a_{k}=0 \Rightarrow\left(x a_{1}\right) \cdot a_{2} \ldots a_{k}=0$ and so $x a_{1}$ is s-nilpotent, i.e. any left multiple of $\mathrm{a}$ is also s-nilpotent.

And hence we 
Note: $\mathrm{a}(\epsilon I)$ would be not s-nilpotent if for any sequence of the type $\left\langle a_{i}\right\rangle, a_{i} \in I$, with $\left(\mathrm{a}=\mathrm{a}_{1}\right)$ we have $a_{1} \cdot a_{2} \ldots a_{k} \neq 0\left(\neq \prod_{i=1}^{k} a_{i}\right)$ whatever be the positive integer $\left.k\right]$

2.1. 5. A sub near-ring $I$ of $N$ is sequentially nilpotent(s-nilpotent) if for each sequence $<a_{i}>, a_{i} \in I$ there is a positive integer $k$ such that $a_{i} \cdot a_{2} \ldots a_{k}=0\left(=\prod_{i=1}^{k} a_{i}\right)$.

Note:

(i) for an s-nilpotent sub near-ring $I$ of $N$, each element of $I$ is s-nilpotent.

(ii) if $I$ is not $s$-nilpotent, then there is a sequence $<a_{i}>, a_{i} \in I$, for each $k, a_{1} \cdot a_{2} \ldots a_{k} \neq 0\left(\neq \prod_{i=1}^{k} a_{i}\right)$, and

2.1.6. $a_{I}(\in I)$ has an infinite sequence if there is a sequence $\left\langle a_{i}>, a_{i} \in I\right.$ such that for each $k$,

$$
a_{1} \cdot a_{2} \ldots a_{k} \neq 0\left(\neq \prod_{i=1}^{k} a_{i}\right) \text {. }
$$

Note:

$I$ is not s-nilpotent, then there is an $a_{l}(\epsilon I)$ such that $a_{l}$ has an infinite sequence.

2.1.7 For $x \in N$ the set $l(x)=\{n \in N \mid n x=0\}$ is the left annihilator of $x$ in $N$.

And this a left ideal of $N$.

2.1.7(a) A near-ring is left Goldie if it satisfies the a.c.c. (ascending chain condition)on its left annihilators and it has no infinite direct sum of left ideals .

2.1.7(b) N is strictly left Goldie if it satisfies the a.c.c. on its left annihilators and it has no infinite independent family of left $\mathrm{N}$-subgroups .

Example 1: $N=\{o, a, b, c\}$ is a near-ring under the operations defined by the following tables.

\begin{tabular}{c|cccc}
+ & 0 & $\mathrm{a}$ & $\mathrm{b}$ & $\mathrm{c}$ \\
\hline 0 & 0 & $\mathrm{a}$ & $\mathrm{b}$ & $\mathrm{c}$ \\
$\mathrm{a}$ & $\mathrm{a}$ & 0 & $\mathrm{c}$ & $\mathrm{b}$ \\
$\mathrm{b}$ & $\mathrm{b}$ & $\mathrm{c}$ & 0 & $\mathrm{a}$ \\
$\mathrm{c}$ & $\mathrm{c}$ & $\mathrm{b}$ & $\mathrm{a}$ & 0
\end{tabular}

(i)

\begin{tabular}{c|cccc}
$\cdot$ & 0 & $\mathrm{a}$ & $\mathrm{b}$ & $\mathrm{c}$ \\
\hline 0 & 0 & 0 & 0 & 0 \\
$\mathrm{a}$ & 0 & 0 & $\mathrm{a}$ & $\mathrm{a}$ \\
$\mathrm{b}$ & 0 & $\mathrm{a}$ & $\mathrm{b}$ & $\mathrm{b}$ \\
$\mathrm{c}$ & 0 & $\mathrm{a}$ & $\mathrm{c}$ & $\mathrm{c}$
\end{tabular}

(ii)

Here we note that $\mathrm{A}=\{0, \mathrm{a}\}, \mathrm{B}=\{0, \mathrm{a}, \mathrm{b}\}$ and $\mathrm{C}=\{0, \mathrm{a}, \mathrm{c}\}$ are subsets of $N$ and $B N \subseteq B, C N \subseteq C$ whereas $N A \subseteq A$ and $A N \subseteq A$. Thus, we define the following

2.1.8 Definitions : A non-empty subset $S$ of a near-ring $N$ is

(i) a right $N$-subset of $N$ if $S N \subseteq S$

(ii) a left $N$-subset of $N$ if $N S \subseteq S$ and

(iii) an invariant subset of $N$ if $N S \subseteq S, S N \subseteq S$.

It is clear that an invariant subset of a near-ring $N$ is a left as well as right $N$-subset of $N$. Moreover, every left (right) $\mathrm{N}$-subset contains the zero element of $N$.

2.1.9 (i) An ideal $I$ of $\mathrm{N}$ is strongly prime if for two non zero invariant subsets $A$ and $B, A B \subseteq I \Rightarrow A \subseteq I$, or $B \subseteq I$.

(ii) A near-ring is strongly prime if (0) is strongly prime.

2.1.10. Definition : If $N$ is a near-ring then the group $(E,+)$ is an $N$-group (near-ring group) $N E$ when there exists a map $N \times E \rightarrow E,(\mathrm{n}, \mathrm{e}) \rightarrow$ ne such that (i) $(\mathrm{n} 1+\mathrm{n} 2) \mathrm{e}=\mathrm{n} 1 \mathrm{e}+\mathrm{n} 2 \mathrm{e}$ 
(ii) $(\mathrm{n} 1 \mathrm{n} 2) \mathrm{e}=\mathrm{n} 1(\mathrm{n} 2 \mathrm{e})$

(iii) 1 . e $=\mathrm{e}$, for all $\mathrm{n} 1, \mathrm{n} 2 \in N$, e $\in E$.

In what follows, $E$ will stand for the near-ring group $N E$.

Clearly near-ring $N$ can always be considered as an $N$ - group. We shall write $N N$ to denote $N$ as an $\mathrm{N}$-group.

Example 2 (Ex.1.18(c) [11] ) : Let $G$ be an additive group and $M(G)$ be a (right) near-ring(of all maps from $G$ to $G)$ then $G$ is an $M(G)$ - group when

$M(G) \times G \rightarrow G$ such that

$(\mathrm{f}, \mathrm{x}) \rightarrow \mathrm{f}(\mathrm{x})$, for $\mathrm{x} \in G, \mathrm{f} \in M(G)$.

Example3 : Every left module $M$ over a ring $\mathrm{R}$ is an $R$-group over the near-ring $R$.

2.1.11. Properties: If $E$ is an $N$-group then

(i) $0 . e=0$ (the first 0 is the zero element of $N$ and the second 0 is the zero element of $E$ ).

(ii) $(-n) e=-n e$ and

(iii) $(n-n 1) e=n e-n 1 e$, for all $e \in E ; n, n 1 \in N$

2.1.12. Definitions: An $N$-group $E$ is said to be an $N$-group with acc on annihilators if any ascending chain

$\operatorname{Ann}(M 1) \subset \operatorname{Ann}(M 2) \subset \operatorname{Ann}(M 3) \subset \ldots$ of annihilators of subsets $M 1, M 2, M 3, \ldots$ of $E$ stops after a finite steps. Similarly, we define an $N$-group $E$ with $d c c$ on annihilators for any descending chain of the type $\operatorname{Ann}(M 1) \supset \operatorname{Ann}(M 2) \supset \operatorname{Ann}(M 3) \supset \ldots$.

\subsection{Essential ideals and essential $N$-subgroups.}

2.2.1. Definitions: Let $A$ and $B$ be two $N$-subgroups of $E$ such that $A \subseteq B$ then $A$ is an essential $N$-subgroup of $B$ (denoted $A \subseteq \mathrm{e} B$ ) if any $N$-subgroup $C(\neq 0)$ of $B$ has non-zero intersection with $A$. when $A \subseteq$ e $B$, we say $B$ is an essential extension of $A$ in $E$. Here an essential left $N$-subgroup $A$ of $N$ will mean an essential $N$-subgroup of $N N$.

An ideal $M$ of $E$ is an essential ideal of $E$ (denoted $M \subseteq \mathrm{e} E$ ) if for any ideal $C(\neq 0)$ of $E$,

$M \cap C \neq(0)$. If a left ideal $A$ of $N$ is an essential ideal of $N N$ then $A$ is an essential left ideal of $N$.

A left N-subgroup of $N$ is weakly essential if for any non zero left ideal $I$ of $N, A \cap I \neq 0$

An essential left ideal $I$ is weakly essential as a left $N$-subgroup. It is to be noted that an essential left $N$ subgroup $A$ of $N$ is also weakly essential. That the converse is not true is shown in example below.

Example4. (H(37), Page 341-342 [11]) : Consider the near-ring $S_{3}=\{0$, a, b, c, x, y) with operation addition [defined in table 1.3 (i)] and multiplication defined by the following table.

$N=\{0, \mathrm{a}, \mathrm{b}, \mathrm{c}, \mathrm{x}, \mathrm{y}\}$ is a near-ring under the operations defined by the following tables.

\begin{tabular}{c|c|c|c|c|c|c|l}
+ & 0 & $\mathrm{a}$ & $\mathrm{b}$ & $\mathrm{c}$ & $\mathrm{x}$ & $\mathrm{y}$ & \\
\hline 0 & 0 & $\mathrm{a}$ & $\mathrm{b}$ & $\mathrm{c}$ & $\mathrm{x}$ & $\mathrm{y}$ & \\
\hline $\mathrm{a}$ & $\mathrm{a}$ & 0 & $\mathrm{y}$ & $\mathrm{x}$ & $\mathrm{c}$ & $\mathrm{b}$ & \\
\hline $\mathrm{b}$ & $\mathrm{b}$ & $\mathrm{x}$ & 0 & $\mathrm{y}$ & $\mathrm{a}$ & $\mathrm{c}$ & \\
\hline $\mathrm{c}$ & $\mathrm{c}$ & $\mathrm{y}$ & $\mathrm{x}$ & 0 & $\mathrm{~b}$ & $\mathrm{a}$ & \\
\hline $\mathrm{x}$ & $\mathrm{x}$ & $\mathrm{b}$ & $\mathrm{c}$ & $\mathrm{a}$ & $\mathrm{y}$ & 0 & \\
\hline $\mathrm{y}$ & $\mathrm{y}$ & $\mathrm{c}$ & $\mathrm{a}$ & $\mathrm{b}$ & 0 & $\mathrm{x}$ &
\end{tabular}

\begin{tabular}{c|c|c|c|c|c|c|l}
$\circ$ & 0 & $\mathrm{a}$ & $\mathrm{b}$ & $\mathrm{c}$ & $\mathrm{x}$ & $\mathrm{y}$ & \\
\hline 0 & 0 & 0 & 0 & 0 & 0 & 0 & \\
\hline $\mathrm{a}$ & 0 & $\mathrm{a}$ & $\mathrm{b}$ & $\mathrm{c}$ & 0 & 0 & \\
\hline $\mathrm{b}$ & 0 & $\mathrm{a}$ & $\mathrm{b}$ & $\mathrm{c}$ & 0 & 0 & \\
\hline $\mathrm{c}$ & 0 & $\mathrm{a}$ & $\mathrm{b}$ & $\mathrm{c}$ & 0 & 0 & \\
\hline $\mathrm{x}$ & 0 & 0 & 0 & 0 & 0 & 0 & \\
\hline $\mathrm{y}$ & 0 & 0 & 0 & 0 & 0 & 0 &
\end{tabular}

Here non-zero left $S_{3}$-subgroups are $\{0, \mathrm{a}\},(0, \mathrm{~b}\},\{0, \mathrm{c}\},\{0, \mathrm{x}, \mathrm{y})$ and $S_{3} .\{0, \mathrm{x}, \mathrm{y})$ and $S_{3}$ are the only nonzero left ideals. This shows that the $\underline{S}_{3}$-subgroup $\{0, \mathrm{x}, \mathrm{y})$ is weekly essential but not an essential left $\underline{S}_{3}$ subgroup.

However, the following example is sufficient to show the existence of near-ring where every weakly essential left $N$-subgroup is also essential.

3.2.16. Example (J(91), Page 343[11]) :

$\mathrm{N}=\{0,1,2,3,4,5,6,7\}$ is a near-ring under addition modulo 8 and multiplication defined by the following table 


\begin{tabular}{l|llllllll}
${ }^{\circ}$ & 0 & 1 & 2 & 3 & 4 & 5 & 6 & 7 \\
\hline 0 & 0 & 0 & 0 & 0 & 0 & 0 & 0 & 0 \\
1 & 0 & 1 & 0 & 3 & 4 & 3 & 0 & 1 \\
2 & 0 & 2 & 0 & 6 & 0 & 6 & 0 & 2 \\
3 & 0 & 3 & 0 & 1 & 4 & 1 & 0 & 3 \\
4 & 0 & 4 & 0 & 4 & 0 & 4 & 0 & 4 \\
5 & 0 & 5 & 0 & 7 & 4 & 7 & 0 & 5 \\
6 & 0 & 6 & 0 & 2 & 0 & 2 & 0 & 6 \\
7 & 0 & 7 & 0 & 5 & 4 & 5 & 0 & 7
\end{tabular}

Here $\{0,4\}$ and $\{0,2,4,6\}$ are the left $N$-subgroup of $N$ whereas the second one is the only non-zero proper left ideal of $N$. Thus each of them is weakly essential and they are essential too.

Example5. ( J (22), Page- 342 - 343 [11] ) :

The group $\mathrm{N}=\{0,1,2,3,4,5,6,7\}$ under addition modulo 8 is an $\mathrm{N}$-group w.r.t. the multiplication defined by the following table

\begin{tabular}{l|llllllll}
$\cdot$ & 0 & 1 & 2 & 3 & 4 & 5 & 6 & 7 \\
\hline 0 & 0 & 0 & 0 & 0 & 0 & 0 & 0 & 0 \\
1 & 0 & 0 & 0 & 2 & 0 & 4 & 4 & 2 \\
2 & 0 & 0 & 0 & 4 & 0 & 0 & 0 & 4 \\
3 & 0 & 0 & 0 & 6 & 0 & 4 & 4 & 6 \\
4 & 0 & 0 & 0 & 0 & 0 & 0 & 0 & 0 \\
5 & 0 & 0 & 0 & 2 & 0 & 4 & 4 & 2
\end{tabular}

$\mathrm{N}$-group $\mathrm{NN}$ has non-trivial $\mathrm{N}$-subgroups $\{0,4\}$ and $\{0,2,4,6\}$. Hence each of them has non-zero intersection with other N-subgroups of $\mathrm{NN}$ and so each of them is an essential N-subgroup of NN. Also $\{0,4\} \subseteq$ e $\{0,2,4$, 6 \} which shows the validity of the following lemma 2.2.2.

2.2.2. Lemma : If $A, B, C$ are $N$-subgroup of $E$ such that $A \subseteq B \subseteq C$ then $A \subseteq e B \subseteq e$ if and only if $A \subseteq e C$.

Proof : Let $P$ be a non-zero $N$-subgroup of $E$ such that $P \subseteq C$. Since $B \subseteq \mathrm{e} C, B \cap P \neq(0)$.

Also, $B \cap \mathrm{P} \subseteq B$ and $A \subseteq \mathrm{e} B$. So $(B \cap P) \cap \mathrm{A} \neq(0)$.

Therefore, $P \cap A \subseteq(B \cap P) \cap A \neq(0)$.

Hence $A \subseteq \mathrm{e} C$

Conversely, let $A \subseteq \mathrm{e} C$. Then $A \cap B \neq(0)$, (for $B \subseteq C$ ).

If $M$ is a non-zero $N$-subgroup of $E$ such that $M \subseteq B \subseteq C$ then, $M$ is a non zero $N$-subgroup of $C$. Since $A \subseteq$ e $C$, it follows that $A \cap M \neq(0)$ which gives $A \subseteq \mathrm{e} B$.

Again, if $H$ is any non-zero $N$-subgroup of $E$ with $H \subseteq C \subseteq E$ then $A \cap H \neq(0)$, (for $A \subseteq$ e $C$ ).

So, $A \subseteq B \Rightarrow(0) \neq A \cap H \subseteq B \cap H$.

Thus, $B \subseteq$ e $C$. //

2.2.3. Lemma : Let $A$ and $B$ be two $N$-subgroups of $E$ such that $B \subseteq e$ A. If $a(\neq 0) \in A$ then there exists an essential $N$-subgroup L of NN such that La $\neq(0)$. 
Proof : Write $L=\{\mathrm{n} \in N \mid$ na $\in B\}$. Clearly, $L \mathrm{a} \subseteq B \subseteq A$ and $N \mathrm{a} \subseteq A$ as $A$ is an $N$-subgroup of $E$, a $\in A$.

Since $1 \in N, N \mathrm{a} \neq(0)$. Again, $B \subseteq \mathrm{e} A$ gives $B \cap N \mathrm{a} \neq(0)$.

Let $(0 \neq) \mathrm{b} \in B \cap \mathrm{Na}$. Then $B=$ na (say) for $\mathrm{n} \in N$. Thus $\mathrm{b}=$ na $\in B$ which gives $\mathrm{n} \in L$. Hence $\mathrm{b}=$ na $\in L \mathrm{a}$.

Therefore, $L a \neq(0)($ for $b \neq 0)$.

Now, let $\mathrm{x}, \mathrm{y} \in L$ then $\mathrm{xa}$, ya $\in B$.

So, $(\mathrm{x}-\mathrm{y}) \mathrm{a}=\mathrm{xa}-\mathrm{ya} \in B$.

$\Rightarrow \mathrm{x}-\mathrm{y} \in L$.

Also, since $B$ is an $N$-group of $E$, for $\mathrm{n} \in N,(\mathrm{nx}) \mathrm{a}=\mathrm{n}($ xa $) \in B$ (for xa $\in B$ )

Therefore, $\mathrm{nx} \in L$.

Thus $L$ is an $N$-subgroup of $N N$.

Again, for an $N$-subgroup $I(\neq 0)$ of $N N$,

$$
\begin{aligned}
& I \mathrm{a}=(0) \\
& \Rightarrow I \mathrm{a} \subseteq B \\
& \Rightarrow I \subseteq L \\
& \Rightarrow L \cap I=I \neq(0)
\end{aligned}
$$

and, $I \mathrm{a} \neq(0)$

$$
\Rightarrow B \cap I \mathrm{a} \neq(0) \text {, (for } I \mathrm{a} \text { is an } N \text {-subgroup of }
$$

$A$ and $\mathrm{B} \subseteq \mathrm{e} A)$.

Now, let $(\neq) \mathrm{x} \in B \cap$ Ia then $\mathrm{x}=\mathrm{b}=\alpha \mathrm{a}$ for

$\mathrm{b} \in B, \alpha \in I$.

Then $\alpha \mathrm{a} \in B$

$$
\begin{aligned}
& \Rightarrow \alpha \in L, \text { (by choice of } L \text { ) } \\
& \Rightarrow \alpha \in L \cap I .
\end{aligned}
$$

Now, $\alpha=0 \Rightarrow \mathrm{x}=0$, a contradiction.

So, $L \cap I \neq(0)$.

Therefore, $\mathrm{L}$ is an essential $N$-subgroup of $N N$ such that $L \mathrm{a} \subseteq B$ and $L \mathrm{La} \neq(0) . / /$

In an $N$-group $E$, the singular $N$-subset of $E$ viz., the subset $\mathrm{Z} 1(E)=\{\mathrm{u} \in E \mid L \mathrm{u}=(0)$, for some essential $N$ subgroup $L$ of $N N$ \} plays an important role in our discussion.

$N$-group $E$ is $N$-non-singular if $\mathrm{Z} 1(E)=0$ and $N$ is left non-singular if $\mathrm{Z} 1(N)=0$. it is to be noted that $\mathrm{Z} 1(E)$ is an $N$-subset of $E$ and $\mathrm{Z} 1(N)$ is an invariant subset of $N$

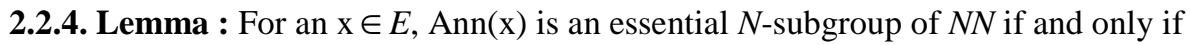
$\mathrm{x} \in \mathrm{Z} 1(E)$.[easy]

2.2.5. Lemma : If I is an $N$-subgroup of $N N$ and for $B \subseteq E$, Ann(B) $\subseteq e$ I and $Z 1(E)=(0)$ then Ann $(B)=I$.

Proof : Let $(0 \neq) \mathrm{x} \in \mathrm{I}$ then by 2.2 .3 , there exists an essential $N$-subgroup $L$ of $N N$ such that $L \mathrm{x} \neq(0), L \mathrm{x}$ $\subseteq$ Ann $(B)$.

$$
\text { So, } \begin{aligned}
(L x) & \mathrm{r}_{\mathrm{E}}(\operatorname{Ann}(B)) \subseteq \operatorname{Ann}(B) \mathrm{r}_{\mathrm{E}}(\operatorname{Ann}(B))=(0) \\
& \Rightarrow L\left(\mathrm{x} \mathrm{r}_{\mathrm{E}}(\operatorname{Ann}(B))=(0) \Rightarrow\left(\mathrm{x} \mathrm{r}_{\mathrm{E}}(\operatorname{Ann}(\mathrm{B}))=(0)[\text { for } \mathrm{Z1}(E)=(0)]\right.\right. \\
& \Rightarrow \mathrm{x} \in \operatorname{Ann}\left(\mathrm{r}_{\mathrm{E}}(\operatorname{Ann}(B))\right)=\operatorname{Ann}(B) \\
& \Rightarrow I \subseteq \operatorname{Ann}(B)
\end{aligned}
$$

Now considering the hypothesis, we get $\operatorname{Ann}(B)=I$. //

2.2.6. Lemma : Let $E$ be with acc on annihilators such that $E$ is $N$-non-singular (i.e.Z1 $(E)=(0))$. If $N$ has no infinite direct sum of left ideals and every essential left ideal of $N$ is an essential $N$-subgroup of $N N$ then $N$ satisfies the dcc on annihilators of subsets of $E$.

Proof : Let $X$ and $Y$ be subsets of $E$ such that $B=\operatorname{Ann}(X)$ and $C=\operatorname{Ann}(Y)$. Thus, $B, C$ are N-subgroups of $N N$.

Now, if $B \subset C$ and $B$ is an essential $N$-subgroup of $C$ then by 2.2.5, $B=C$ as $B=\operatorname{Ann}(X)$. Hence $B$ is not an essential $N$-subgroup of $C$. So, there exists an $N$-subgroup $D(\neq 0)$ of $N N$ such that $D \subseteq C, B \cap D=(0)$. 
Let $A_{1} \supset A_{2} \supset A_{3} \supset \ldots$ be a strictly descending chain of annihilators of subsets of $E$. Since $\mathrm{A}_{1} \supset \mathrm{Ai}_{+1}$, by the above argument, there exists an $N$-subgroup $P i \quad(\neq 0)$ of $N N$ such that $P_{\mathrm{i}} \subseteq A_{\mathrm{i}}$ and $A_{\mathrm{i}+1} \cap P_{\mathrm{i}}=(0)$ (i)

Consider $M=\left\{X_{\mathrm{m}}\right\}$, the family of all left ideals of $N$ such that $A \mathrm{i}+1 \cap X \mathrm{~m}=(0)$. The union of each chain of $M$ is again a left ideal in $M$ and satisfies the condition $A \mathrm{i}+1 \cap X \mathrm{~m}=(0)$. Thus, by Zorn's Lemma $M$ has a maximal element $X \mathrm{i}$ (say) such that $A \mathrm{i}+1 \cap X \mathrm{i}=(0)$

Again, $A \mathrm{i}+1$ and $X \mathrm{i}$ being left ideals of $N, A \mathrm{i}+1+X \mathrm{i}$ is also a left ideal of $N$.

Now, let $V$ be a left ideal of $N$ such that $(A \mathrm{i}+1+X \mathrm{i}) \cap V=(0)$.

Now, ai+1 $=x i+v$, for some $a i+1 \in A i+1, x i \in X i, v \in V$.

$$
\begin{aligned}
& \Rightarrow \mathrm{v}=-\mathrm{xi}+\mathrm{ai}+1 \in X \mathrm{i}+\mathrm{Ai}+1 \subseteq A \mathrm{i}+1+\mathrm{xi} \\
& \Rightarrow \mathrm{v} \in(A \mathrm{i}+1+X \mathrm{i}) \cap V=(0) \\
& \Rightarrow \mathrm{ai}+1=\mathrm{xi} \in A \mathrm{i}+1 \cap X \mathrm{i}=(0) \\
& \Rightarrow A \mathrm{i}+1 \cap(X \mathrm{i}+V)=(0)
\end{aligned}
$$

Since $X \mathrm{i}$ is maximal with condition $A \mathrm{i}+1 \cap X i=(0)$, it follows that $X \mathrm{i}+V=X \mathrm{i}$ as $X \mathrm{i} \subseteq X \mathrm{i}+V$. This gives $V \subseteq$ $X \mathrm{i}$ and so $V=V \cap X \mathrm{i} \subseteq V \cap(A \mathrm{i}+1+X i)=(0)$.

Thus, $A \mathrm{i}+1+X \mathrm{i}$ is an essential left ideal of $N$ such that $A i+1 \cap X \mathrm{i}=(0)$ and the assumed hypothesis gives that $A i+1+X \mathrm{i}$ is an essential N-subgroup of $N N$. And so for $P \mathrm{i}$, chosen above, $P i \cap(A \mathrm{i}+1+X \mathrm{i}) \neq(0)$.

Suppose, $\alpha \in(P i)=\mathrm{ai}+1+\mathrm{xi}$, for $\alpha \mathrm{i} \in P \mathrm{i}$, ai+1 $\in A \mathrm{i}+1$, xi $\in \mathrm{Xi}$.

Then, $\mathrm{xi}=-\mathrm{ai}+1+P \mathrm{i} \subseteq \in A \mathrm{i}+1+P \mathrm{i} \subseteq A \mathrm{i}+\mathrm{Pi}$, for $A \mathrm{i}+1 \subseteq A \mathrm{i}$. So, xi $\in A \mathrm{i}$ (for $P \mathrm{i} \subseteq A \mathrm{i}$ ) which gives $\mathrm{xi} \in A \mathrm{i}$ $\cap X \mathrm{i}$.

Now, if $\mathrm{xi}=0$ then $P \mathrm{i} \in A \mathrm{i}+1$ which gives $P \mathrm{i} \in A \mathrm{i}+1$. $P \mathrm{i}=(0)$. So, $P \mathrm{i}=0$.

Therefore, $P \mathrm{i} \cap(A \mathrm{i}+1+X \mathrm{i})=(0)$ and this is a contradiction. Hence $\mathrm{xi} \neq 0$ and therefore

$A \mathrm{i} \cap X \mathrm{i} \neq(0)$.

Let $C \mathrm{i}=A \mathrm{i} \cap X \mathrm{i}$, a non-zero left ideal of $N$.

Then, $C \mathrm{i} \cap A \mathrm{i}+1=(A \mathrm{i} \cap X \mathrm{i}) \cap A \mathrm{i}+1$

$$
\begin{aligned}
& =(A \mathrm{i}+1 \cap A \mathrm{i}) \cap X \mathrm{i} \\
& =A i+1 \cap X \mathrm{i}, \quad(\text { as } A \mathrm{i} \supset A \mathrm{i}+1) \\
& =(0), \quad[\text { by (ii) }]
\end{aligned}
$$

Therefore, when $A i \supset A \mathrm{i}+1$, we get a non-zero ideal $C \mathrm{i}=A \mathrm{i} \cap X \mathrm{i}$ such that $C i \cap A \mathrm{i}+1=(0)$

Now, for different values of $\mathrm{i}$, we get an infinite family $\{C 1, C 2, C 3, \ldots\}$ of non-zero left ideals of $\mathrm{N}$ such that (iii) holds.

Also, $C \mathrm{i}=A \mathrm{i} \cap X \mathrm{i} \subseteq A \mathrm{i}$

Therefore, $C 1 \cap C 2 \subseteq C 1 \cap A 2=(0)$, [by (iii) and (iv)]

Again, $C 1 \cap(C 2+C 3) \subseteq C 1 \cap(A 2+A 3)$, [by (iv)]

$\subseteq C 1 \cap A 2$, as $A 2 \supset A 3$

$=(0), \quad[$ by (iii) $]$

$\Rightarrow C 1 \cap(C 2+C 3)=(0)$

And if $\mathrm{x} \in C 2 \cap(C 1+C 3)$ then

$\mathrm{x}=\mathrm{c} 2=\mathrm{c} 1+\mathrm{c} 3$, for $\mathrm{ci} \in C \mathrm{i}, \mathrm{i}=1,2,3$.

$\Rightarrow \mathrm{c} 1=\mathrm{c} 2-\mathrm{c} 3 \in \mathrm{C} 2+\mathrm{C} 3$

So, c1 $\in C 2 \cap(C 2+C 3)=(0), \quad[$ by $(v)]$

$\Rightarrow \mathrm{c} 1=0$ and $\mathrm{c} 2=\mathrm{c} 3 \in \mathrm{C} 3$.

$\Rightarrow C 2 \in C 2 \cap C 3 \subseteq C 2 \cap A 3=(0)$,

$\Rightarrow \mathrm{c} 2=0$ and hence $C 2 \cap(C 1+C 3)=(0)$.

[by (iii) and (iv)]

Similarly, $C 3 \cap(C 1+C 2)=(0)$. Thus $C 1 \oplus C 2 \oplus C 3$ is a direct sum of non-zero left ideals of $\mathrm{N}$. 
Proceeding in this way, we find an infinite direct sum $C 1 \oplus C 2 \oplus C 3 \oplus \ldots \ldots$ of nonzero left ideals of $N$. This goes against our hypotheses and hence there exists a $\mathrm{t} \in Z^{+}$such that $A \mathrm{t}=A \mathrm{t}+1=A \mathrm{t}+2=\ldots$ Therefore, $N$ satisfies the dcc on annihilators of subset of $E$. //

2.2.7. Lemma : $Z_{1}(N)(=\{x \in N \mid A x=(0)$, for some essential left $N$-subgroup A of $N\})$ is an invariant subset of N.

Proof : Let $\mathrm{x} \in \mathrm{Z}_{1}(N)$. Then $A \mathrm{x}=(0)$, for some essential left $N$-subgroup $A$ of $N$. So, by 2.2 .3 , for any n

$(\neq 0) € N$ there exists an essential left $N$-subgroup $L$ of $N$ such that

Ln $\subseteq A, L \mathrm{n} \neq(0)$.

This gives, $L(\mathrm{nx})=(L \mathrm{n}) \mathrm{x} \subseteq A \mathrm{x}=(0)$

$\mathrm{nx} \in \mathrm{Z}_{1}(N)$.

And, $A(\mathrm{xn})=(A \mathrm{x}) \mathrm{n}=(0)$

$\mathrm{xn} \in \mathrm{Z}_{1}(N) / /$

2.2.8. Lemma : A strongly semiprime near-ring $N$ with acc on left annihilators has no non-zero nil left $N$ subset of $N$.

Proof : Let $A$ be any non-zero left $N$-subset of $N$. Since $N$ satisfies the acc on left annihilators, we can choose a

$(\neq 0) \in A$ with $1($ a) as large as possible.

Now, $\mathrm{a} N \mathrm{a}=(0)$

$\Rightarrow(N a)^{2}=(N a)(N a)=N(a N a)=(0)$

And $N$ a being a non-zero left $N$-subset of $N(1 € N, \mathrm{a} \neq 0)$, we meet a contradiction to 3.2.5. [N being strongly semi prime has no non-zero nilpotent left or right $N$-subset]

So, $\mathrm{a} N \mathrm{Na} \neq(0)$.

Let $\mathrm{x} \in N$ be such that axa $\neq 0$

Now, $x a \neq 0$ (otherwise axa $=0$ )

$\mathrm{x} \neq 1(\mathrm{a})$

Again, $\mathrm{z} \in 1(\mathrm{a}) \Rightarrow \mathrm{za}=0$

$$
\begin{aligned}
& \Rightarrow z(a x a)=(z a) x a=0 \\
& \Rightarrow z \in 1(\text { axa }) \\
& 1(a) \in 1 \text { (axa) }
\end{aligned}
$$

But 1(a) being maximal, 1(axa) = 1(a)

So, $x^{2} \notin 1($ axa $)$

$$
\begin{aligned}
& \Rightarrow x(a x a)=0 \\
& \Rightarrow(x a)^{2}=0 \\
& \Rightarrow(x a x) a=0 \\
& \Rightarrow x a x 1(a)=1(a x a) \\
& \Rightarrow(x a x)(x a x)=0 \\
& \Rightarrow(x a)^{3} \neq 0 \text { and so on. }
\end{aligned}
$$

Thus, (xa) ${ }^{\mathrm{t}} \neq 0$, for any $\mathrm{t} \in Z^{+}$.

Therefore, $A$ possesses a non-zero non nilpotent element xa. So $A$ is not nill.

Hence $N$ does not have any non-zero nil left $N$-subset of $N$. I

2.2.9. Lemma : If $N$ is a strongly semiprime near-ring with acc of left annihilators then $N$ is left non-singular.

Proof : Being $N$ acc with acc on left annihilators, $\mathrm{Z}_{1}(N)$ is a nil invariant subset of $N$ and by above it follows that $\mathrm{Z}_{1}(N)=0$. Thus the result follows.

Again N being strictly left Goldie, it is left Goldie. So it has no infinite direct sum of left ideals. And therefore as a special case of 2.2.6, we get the following ( [5], Nat, Acad,Sci. Letters.)

2.2.10. Theorem : If in a strongly semiprime strictly left Goldie near-ring $N$, every weakly essential left $N$ subgroup of $N$ is also essential, then $N$ satisfies the dcc on left annihilators.

And now we get the following effective result for our purpose. 
2.2.11 Corollary: In A strongly semiprime strictly left Goldie near-ring $N$, if every weakly essential left $N$ subgroup of $N$ is also essential, then $N$ satisfies the a.c.c. on left as well as right annihilators.

\section{Main Result}

3.1. Theorem

Suppose $N$ satisfies the acc on left annihilators and I is a nil subring of $N$ and I is not left s-nilpotent. Then there exists a sequence $\left\langle a_{i}\right\rangle, a_{i} \in N$ such that $N a_{i} \neq 0$ and the family $\left\{N a_{i}\right\}$ is an independent family, or the sum $N a_{1}+N a_{2}+\ldots$ is direct.

Proof: It is assumed that $I$ is not s-nilpotent. Then there is an element $y \in I$ such that $y$ has an infinite chain $<y_{i}>, y_{i} \in I$ with $\left(y y_{1} y_{2} \ldots y_{k-1} y_{k} \neq 0, \forall k\right)$. We now consider the following

We have $y_{l} \in I$ such that $y y_{1} \neq 0$,

$y_{2} \in I$ such that $y y_{1} y_{2} \neq 0$

$\mathrm{y}_{3} \in I \quad$ such that $\mathrm{yy}_{1} \mathrm{y}_{2} \mathrm{y}_{3} \neq 0$ ........ And so on.

So we clearly have the following possibilities

There exists $x \in I$ such that $y x \neq 0$ ( for example $y_{1}$ is such an element, and we may have more than one such element!)

There exists $x \in I N$ such that $y y_{1} x \neq 0$ ( for example $y_{2}$ is such an element, and we may have more than one such element!)

..... etc

Thus it is possible to define a sequence $y_{1}, y_{2},$. of $N$ such that

$K_{1}=\{x \in I \mid y x$ has an infinite chain $\}$

$K_{2}=\left\{x \in I \mid y y_{1} x\right.$ has an infinite chain $\}$

$K_{3}=\left\{x \in I \mid y y_{1} y_{2} x\right.$ has an infinite chain $\}$

In general

$K_{n}=\left\{x \in I \mid y_{1} y_{2} . . y_{n-2} y_{n-1} y x\right.$ has an infinite chain $\}$

As $N$ satisfies the acc on left annihilators, now we consider the maximal element $1\left(\mathrm{y}_{\mathrm{n}}\right)$ with $y_{\mathrm{n}} \in K_{\mathrm{n}}$.

We now claim

For each i,$l\left(y_{\mathrm{i}}\right)=l\left(y_{\mathrm{i}} \cdot y_{\mathrm{i}+1 \ldots} y_{\mathrm{i}+\mathrm{j}}\right) \quad($ for all $j \geq 1)$

In particular note that $l\left(y_{1}\right)=l\left(y_{1} y_{2} y_{3}\right),(i=1, j=2)$

As $x \in l\left(y_{1}\right) \Rightarrow x y_{1}=0$, clearly $x y_{1} y_{2} y_{3}=0$ which gives

easily, $x \in l\left(y_{1} y_{2} y_{3}\right)$ i.e. $\Rightarrow l\left(y_{1}\right) \subseteq l\left(y_{1} y_{2} y_{3}\right)$

Now $y_{1} \in K_{1}$ with $l\left(y_{1}\right)$ maximum

$y_{2} \in K_{2}\left[=\left\{x \in I \mid y y_{1} x\right.\right.$ has an infinite chain $\left.\}\right]$ with $l\left(y_{2}\right)$ maximum

$y_{3} \in K_{3} \quad\left[=\left\{x \in I \mid y y_{1} y_{2} x\right.\right.$ has an infinite chain $\left.\}\right]$ with $l\left(y_{3}\right)$ maximum

so, $y y_{1} y_{2} y_{3}$ has an infinite chain. And

i.e., $x\left(=y_{1} y_{2} y_{3}\right) y$ i.e., $x y$ has an infinite chain ( here, $x \in N$ )

so, $x \in K_{1}$ i.e., $y_{1} y_{2} y_{3} \in K_{1}$

And therefore, $l\left(y_{1} y_{2} y_{3}\right) \subseteq l\left(y_{1}\right)$

[ using the maximality of $l\left(y_{1}\right)$ ]

Now $*$ gives and $* *$ give

$l\left(y_{1} y_{2} y_{3}\right)=l\left(y_{1}\right)$

We now set

$a_{1}=y y_{1}, a_{2}=y y_{1} y_{2}, a_{3}=y y_{1} y_{2} y_{3}$, etc.

In general, $a_{\mathrm{n}}=y y_{1} \ldots y_{\mathrm{n}-1} y_{\mathrm{n}}$

Suppose, $a_{1} y_{1} \neq 0$, then $a_{1} y_{1} y_{2} \neq 0$, for if $a_{1} y_{1} y_{2}=0$, then $a_{1} \in l\left(y_{1} y_{2}\right) \Rightarrow y_{1} a_{1}=0\left[\right.$ as $\left.l\left(y_{1}\right)=l\left(y_{1} y_{2}\right)\right]$, hence, $a_{1} y_{1}=0$.

Similarly, we have, $a_{1} y_{1} y_{2} y_{3} \neq 0$ for if $a_{1} y_{1} y_{2} y_{3}=0 \Rightarrow a_{1} \in l\left(y_{1} y_{2} y_{3}\right)=l\left(y_{1}\right)$

Claim $a_{\mathrm{n}} y_{1}=0$

Suppose $a_{\mathrm{n}} y_{1} \neq 0$, for some $n$.

we now consider the case for any $k$. 
$a_{\mathrm{n}} y_{1} y_{2} \ldots y_{\mathrm{k}-1} y_{\mathrm{k}} \neq 0$, for $l\left(y_{1}\right)=l\left(y_{1} y_{2} \ldots y_{\mathrm{k}-1} y_{\mathrm{k}}\right)$

$a_{\mathrm{n}} y_{1} y_{2} \ldots y_{\mathrm{k}-1} y_{\mathrm{k}}=0 \Rightarrow a_{\mathrm{n}} \in l\left(y_{1} y_{2} \ldots y_{\mathrm{k}-1} y_{\mathrm{k}}\right)=l\left(y_{1}\right)$

$\Rightarrow a_{\mathrm{n}} y_{1}=0$, a contradiction

so, $a_{\mathrm{n}} y_{1} y_{2} \ldots y_{\mathrm{k}-1} y_{\mathrm{k}} \neq 0$, i.e. $\left(y y_{1} y_{2} \ldots y_{\mathrm{n}}\right)\left(y_{1} y_{2} \ldots y_{\mathrm{k}-1} y_{\mathrm{k}}\right) \neq 0$

$\Rightarrow\left(y y_{1} y_{2} \ldots y_{\mathrm{n}} y_{1}\right)\left(y_{2} y_{3} \ldots y_{\mathrm{k}}\right) \neq 0$

And this gives $\mathrm{y}_{2} \mathrm{y}_{3} \ldots$ forms a chain for $\mathrm{y}_{1} \mathrm{y}_{2} \ldots \mathrm{y}_{\mathrm{n}} \mathrm{y}_{1}=\mathrm{y}\left(\mathrm{y}_{1} \mathrm{y}_{2} \ldots \mathrm{y}_{\mathrm{n}} \mathrm{y}_{1}\right)=\mathrm{yx}, \mathrm{x} \in \mathrm{N}$

$\therefore \mathrm{x}=\mathrm{y}_{1} \mathrm{y}_{2} \ldots \mathrm{y}_{\mathrm{n}} \mathrm{y}_{1} \in \mathrm{K}_{1}(\subseteq I)$ and since, in $\left.\mathrm{K}_{1}, \mathrm{l}_{(} \mathrm{y}_{1}\right)$ is maximum,

$1\left(\mathrm{y}_{1} \ldots \mathrm{y}_{\mathrm{n}} \ldots \mathrm{y}_{1}\right) \subseteq 1\left(\mathrm{y}_{1}\right)$ and if $\alpha \in 1\left(\mathrm{y}_{1}\right), \alpha \mathrm{y}_{1}=0 \Rightarrow \alpha \mathrm{y}_{1} \mathrm{y}_{\mathrm{n}} \ldots \mathrm{y}_{1}=0$

$\Rightarrow \alpha \in 1\left(\mathrm{y}_{1} \mathrm{y}_{\mathrm{n}} \ldots \mathrm{y}_{1}\right) \Rightarrow \mathrm{l}\left(\mathrm{y}_{1}\right) \subseteq 1\left(\mathrm{y}_{1} \mathrm{y}_{\mathrm{n}} \ldots \mathrm{y}_{1}\right) \Rightarrow \mathrm{l}\left(\mathrm{y}_{1}\right)=1\left(\mathrm{y}_{1} \mathrm{y}_{\mathrm{n}} \ldots \mathrm{y}_{1}\right) \ldots(\alpha)$

since, $\mathrm{y}_{1}, \mathrm{y}_{2}, \ldots, \mathrm{y}_{\mathrm{n}} \in I, \mathrm{y}_{\mathrm{n}} . \mathrm{y}_{2} \cdot \mathrm{y}_{1} \in I$,

$\because \mathrm{y}_{\mathrm{n}} \cdot \mathrm{y}_{2} \cdot \mathrm{y}_{1} \in I($ nil $), \mathrm{y}_{1} \cdot . \mathrm{y}_{2} \cdot \mathrm{y}_{\mathrm{n}}$ is nilpotent (since, $\left.\mathrm{y}_{1} \cdot . \mathrm{y}_{2} \cdot \mathrm{y}_{\mathrm{n}} \in I-n i l\right)$, say $\left(\mathrm{y}_{1} \cdot . \mathrm{y}_{2} \cdot \mathrm{y}_{\mathrm{n}}\right)^{2}=0$

(note, here nilpotency of $I$ is used!!)

and therefore, $\left(\mathrm{y}_{1} \mathrm{y}_{2} \ldots \mathrm{y}_{\mathrm{n}}\right)\left(\mathrm{y}_{1} \mathrm{y}_{2} \ldots \mathrm{y}_{\mathrm{n}}\right)=0 \Rightarrow\left(\mathrm{y}_{1} \mathrm{y}_{2} \ldots \mathrm{y}_{\mathrm{n}}\right)\left(\mathrm{y}_{1} \mathrm{y}_{2} \ldots \mathrm{y}_{\mathrm{n}}\right) \mathrm{y}_{1}=0$

$\Rightarrow\left(\mathrm{y}_{1} \mathrm{y}_{2} \ldots \mathrm{y}_{\mathrm{n}}\right)\left(\mathrm{y}_{1} \mathrm{y}_{2} \ldots \mathrm{y}_{\mathrm{n}} \mathrm{y}_{1}\right)=0 \Rightarrow\left(\mathrm{y}_{1} \mathrm{y}_{2} \ldots \mathrm{y}_{\mathrm{n}}\right) \in \mathrm{l}\left(\mathrm{y}_{1} \mathrm{y}_{\mathrm{n}} \ldots \mathrm{y}_{1}\right)=1\left(\mathrm{y}_{1}\right)[$ by $(\alpha)]$

$\Rightarrow \mathrm{y}_{1} \mathrm{y}_{2} \ldots \mathrm{y}_{\mathrm{n}} \mathrm{y}_{1}=0 \Rightarrow \mathrm{yy}_{1} \mathrm{y}_{2} \ldots \mathrm{y}_{\mathrm{n}} \mathrm{y}_{1}=0 \Rightarrow\left(\mathrm{yy}_{1} \mathrm{y}_{2} \ldots \mathrm{y}_{\mathrm{n}}\right) \mathrm{y}_{1}=0 \Rightarrow \mathbf{a}_{\mathbf{n}} \mathbf{y}_{\mathbf{1}}=\mathbf{0}$.

Similarly, for all $\mathrm{i}, \mathrm{a}_{\mathrm{n}} \mathrm{y}_{\mathrm{i}}=0$, for all $\mathrm{n} \geq \mathrm{i}$.

Now we show that

(i) $\quad$ all $N \mathrm{a}_{\mathrm{i}} \neq 0$ for $1 \in \mathrm{N}$

(ii) to show that the sum $N \mathrm{a}_{1}+\mathrm{Na}_{2}+\ldots$ is direct or

the family $\mathrm{Na}_{1}, \mathrm{Na}_{2}$, is an independent family.

We first show that $N \mathrm{a}_{1} \cap N \mathrm{a}_{2}=0$.

That is if $\mathrm{n}_{1} \mathrm{a}_{1}=\mathrm{n}_{2} \mathrm{a}_{2}$ for some $\mathrm{n}_{1}, \mathrm{n}_{2} \in N$, then $\mathrm{n}_{1} \mathrm{a}_{1}=\mathrm{n}_{2} \mathrm{a}_{2}=0$

Now we note that $\mathrm{y} \in I$ is such that $\mathrm{y}$ has an infinite sequence and choose $1(\mathrm{y})$ to be maximum. And $\mathrm{y}_{1} \mathrm{y}$ is such that $\mathrm{y}_{1} \mathrm{y}$ has an infinite chain with $\mathrm{l}\left(\mathrm{y}_{1}\right)$ is maximum,

similarly, $\mathrm{yy}_{1} \mathrm{y}_{2}$ is such that $\mathrm{y}_{1} \mathrm{y}_{2}$ has an infinite chain with $1\left(\mathrm{y}_{2}\right)$ is maximum,

And therefore, $1\left(\mathrm{yy}_{1} \mathrm{y}_{2}\right) \subseteq 1(\mathrm{y}) \ldots$

But clearly we have, $1(\mathrm{y}) \subseteq 1\left(\mathrm{yy}_{1} \mathrm{y}_{2}\right) \ldots \ldots$

Therefore, $1(\mathrm{y})=1\left(\mathrm{y}_{1} \mathrm{y}_{2}\right)$. And hence,

$\mathrm{n}_{1} \mathrm{a}_{1}=\mathrm{n}_{2} \mathrm{a}_{2} \Rightarrow \mathrm{n}_{1} \mathrm{a}_{1} \mathrm{y}_{2}=\mathrm{n}_{2} \mathrm{a}_{2} \mathrm{y}_{2}=0\left(\right.$ as $\left.\mathrm{a}_{2} \mathrm{y}_{2}=0\right)$

$\Rightarrow \mathrm{n}_{1} \mathrm{yy}_{1} \mathrm{y}_{2}=0\left(\right.$ as $\left._{1}=\mathrm{yy}_{1}\right) \Rightarrow \mathrm{n}_{1} \in \mathrm{l}\left(\mathrm{yy}_{1} \mathrm{y}_{2}\right)=\mathrm{l}(\mathrm{y}) \Rightarrow \mathrm{n}_{1} \mathrm{y}=0$

$\Rightarrow \mathrm{n}_{1} \mathrm{yy}_{1}=0 \Rightarrow \mathrm{n}_{1} \mathrm{a}_{1}=0$

$\Rightarrow$ i.e. $\mathrm{n}_{1} \mathrm{a}_{1}=\mathrm{n}_{2} \mathrm{a}_{2}=0$, thus $N \mathrm{a}_{1} \cap N \mathrm{a}_{2}=0$, i.e. $N \mathrm{a}_{1}+N \mathrm{a}_{2}$ is direct.

Similarly, the sum $\mathrm{Na}_{1}+\mathrm{Na}_{2}+\ldots$ is direct.

Now we'll show that

\subsection{Theorem}

If $\left\{S_{i}=a_{j} \mid j \geq i\right\}$ then $r\left(S_{i}\right), i=1,2, .$. form a strictly ascending chain of right annihilators.

Proof: Here, $S_{1}=\left\{a_{j} \mid a_{j} \geq 1\right\}=\left\{a_{1}, a_{2}, a_{3}, \ldots\right\}, S_{2}=\left\{a_{j} \mid a_{j} \geq 2\right\}=\left\{a_{2}, a_{3}, a_{4}, \ldots\right\}$

$S_{3}=\left\{a_{j} \mid a_{j} \geq 3\right\}=\left\{a_{3}, a_{4}, a_{5}, \ldots\right\}$

$S_{i}=\left\{a_{j} \mid a_{j} \geq i\right\}=\left\{a_{i}, a_{i+1}, a_{i+2}, \ldots\right\}$

Now, $S_{1} \mathrm{x}=0 \Rightarrow \mathrm{a}_{1} \mathrm{x}=\mathrm{a}_{2} \mathrm{x}=\mathrm{a}_{3} \mathrm{x}=\ldots=0$

and this $\Rightarrow a_{2} x=a_{3} x=. .=0$

$\Rightarrow S_{2} \mathrm{x}=0 \Rightarrow r\left(S_{1}\right) \subseteq r\left(S_{2}\right)$,

similarly, $r\left(S_{1}\right) \subseteq r\left(S_{2}\right) \subseteq r\left(S_{3}\right) \subseteq r\left(S_{4}\right) \ldots \ldots$

Again, $\mathrm{a}_{2} \mathrm{y}_{2}=0 \mathrm{a}_{3} \mathrm{y}_{2}=\mathrm{a}_{4} \mathrm{y}_{2}=\ldots=0$ but, $\mathrm{a}_{1} \mathrm{y}_{2} \neq 0$ (for $\mathrm{y}_{1} \mathrm{y}_{2} \neq 0$ ). 
Hence, $\mathrm{y}_{2} \in \mathrm{r}\left(\mathrm{S}_{2}\right)$ and $\mathrm{y}_{2} \notin \mathrm{r}\left(\mathrm{S}_{1}\right)$. And therefore, $r\left(S_{1}\right) \subset r\left(S_{2}\right)$ similarly, $r\left(S_{2}\right) \subset r\left(S_{3}\right)$, i.e. $r\left(S_{1}\right) \subset r\left(S_{2}\right) \subset r\left(S_{3}\right) \subset \ldots$

is a strictly ascending infinite chain of left annihilators.//

\section{Main result}

Now we prove the main results that we are aiming for.

3.3 Theorem: If I is not nilpotent, then I is not s-nilpotent.

[Note: so, if $I$ is s-nilpotent the $I$ is nilpotent, and if $I$ is nil then it is s-nilpotent]

Proof: We consider $I, I^{2}, I^{3}, \ldots$ and clearly, we have

$I \supseteq I^{2} \supseteq I^{3} \supseteq \ldots$ and therefore,

$l(I) \subseteq l\left(I^{2}\right) \subseteq l\left(I^{3}\right) \subseteq \ldots$ and

by acc on left annihilators, we have an integer, say k, such that

$l\left(I^{k}\right)=l\left(I^{s}\right)$ for all

$\mathrm{s} \geq \mathrm{k}$.

So if we set $\mathrm{K}=I^{\mathrm{k}}$, then $l(K)=l\left(K^{2}\right)$. And $\mathrm{K}^{2} \neq 0\left(\right.$ for if $\mathrm{K}^{2}=0$, then $I$ appears as nilpotent, which is not true.

And thus $K^{2} \neq 0$, that gives an $\mathrm{x}_{1} \in K$ such that $\mathrm{x}_{1} K \neq 0$.

And this gives $\mathrm{x}_{1} K^{2} \neq 0$. For if $\mathrm{x}_{1} K^{2}=0$,

then $\mathrm{x}_{1} \in \mathrm{l}\left(K^{2}\right)=1(K) \Rightarrow \mathrm{x}_{1} K=0$, a contradiction.

Now, $\mathrm{x}_{1} \mathrm{~K}^{2} \neq 0 \Rightarrow \mathrm{x}_{2} \in K$ such that $\mathrm{x}_{2} \mathrm{x}_{1} K \neq 0$. And so on.

Thus we get $\mathrm{x}_{3}, \mathrm{x}_{4} \ldots$ are such that each of $\mathrm{x}_{1}, \mathrm{x}_{1} \mathrm{x}_{2}, \mathrm{x}_{1} \mathrm{x}_{2} \mathrm{x}_{3}, \ldots$ is non zero .

Therefore, the sequence $\left\langle x_{n}\right\rangle$ is such that

each $\mathrm{x}_{\mathrm{i}} \in I$ and $\ldots . . \mathrm{x}_{\mathrm{k}} . . \mathrm{x}_{2} \mathrm{x}_{1} \neq 0$.

Hence, $I$ is not s-nilpotent.//

3.4 Theorem: If $N$ is a strongly semiprime strictly left Goldie near-ring where every weakly essential left $N$ subgroup of $N$ is also essential, then the each nil-subring of $N$ is nilpotent.

Proof: Let $I$ be a nil subnear-ring of $N$. (to prove that $I$ is nilpotent!).

Suppose, $I$ is not nilpotent. Then by above $I$ is not s-nilpotent.

Then we have an infinite sequence $\mathrm{a}_{1}, \mathrm{a}_{2}, \mathrm{a}_{3}, \ldots$ in $N$ such that each $N \mathrm{a}_{\mathrm{i}}$ is non zero and their sum is direct, and the chain

$r\left(S_{1}\right) \subset r\left(S_{2}\right) \subset r\left(S_{3}\right) \subset \ldots$. is a strictly infinite ascending chain of right annihilators. Now as $N$ is with the acc on right annihilators ( 2.2.11 Corollary) such a sequence is not possible. Thus, I must be nilpotent.

The paper is written under emeritus fellowship

\section{Acknowledgement}

\section{REFERENCES}

[1]. Chowdhury, K.C. : Goldie near-rings, bull. Cal. Math.soc.80(1988), no. 4,261-269

[2]. Goldie modules, IJPAM 19(7), 641-652, july 1988

[3]. Goldie M-group, Australian math. Soc.

[4]. Goldie theorem analogue for Goldie near-rings,IJPAM,20(2),141- 149,Feb,1989

[5]. A note on regular left Goldie near-ring, nat.acad. sci. letters,vol.12, no.(1989),433-435

[6]. FSD N-subgroup with acc on annihilators IJPAM 24(2),747-755,Dec1993

[7]. N-groups with acc on annihilators - some topological properties, Mathematica Pannonica, 15/1, (2004), 65-84)

[8]. Some Aspects of Artinian (Noetherian ) Part of a Goldie Ring and its Topological

[9]. Relevance, Southeast Asian Bulletin of Mathematics, 32:8(2008), 43-853

[10]. Wreath Sum of Near-rings and Near-ring Groups, Southeast Asian Bulletin of Mathematics, 36(2012), 169-18

[11]. Goldie, A.W. : The structure of prime rings under ascending chain conditions, proc. London math sc.(3) 8 (1958), 589-608

[12]. Semi-prime rings with maximum conditions proc.lond. math. Ssoc. 10(1960), 201-220

[13]. Lanski, C. :Nil sub rings of Goldie rings are nilpotent.,Canadian j. math. 21(1969) 904-907

[14]. Pilz, G : Near-rings( the theory and its applications) : North Holland Publ. comp.1977 